\title{
Metodologías didácticas en formación en línea: el caso de la Universidad Internacional de Valencia
}

\section{Didactic methodologies in online training: the case of the Valencian International University}

\author{
Ernesto Colomo Magaña ${ }^{1}$, Vicente Gabarda Méndez ${ }^{2}$, Ana Rodríguez Martín ${ }^{3}$ \\ ${ }^{1}$ Universidad Internacional de Valencia, España (ernesto.colomo@campusviu.es) \\ ${ }^{2}$ Universidad Internacional de Valencia, España (vgabarda@universidadviu.com) \\ ${ }^{3}$ Universidad Internacional de Valencia, España (arodriguez@universidadviu.com)
}

Recibido el 11 de junio de 2018; revisado el 24 de septiembre de 2018; aceptado el 24 de septiembre de 2018; publicado el 1 de diciembre de 2018

\section{RESUMEN:}

En todo proceso de enseñanza-aprendizaje, las metodologías determinan las formas de trabajar en los procesos educativos para que el alumnado adquiera los correspondientes aprendizajes. Se trata de una realidad que marca el acto educativo, existiendo diferentes modalidades y roles por parte del docente en el desarrollo de las mismas. En el contexto de la formación en línea, teniendo a la Universidad Internacional de Valencia como referente, el objetivo de este estudio ha sido analizar el uso de las metodologías que se aplican en un contexto de formación en línea por parte de 106 profesores que impartieron docencia en el área de educación durante el curso 2017-2018. La información se recopiló mediante un cuestionario autoadministrado que se realizó ad hoc para este estudio centrado en cuatro ítems: metodologías implementadas en la institución, frecuencia de uso, rol del docente en la aplicación de las metodologías y tipo de conocimiento que se favorece en el estudiante. Los resultados muestran que la mayoría de las metodologías se utilizan en el aprendizaje en línea de manera generalizada, salvo la de Hand-On. En ellas, el rol del docente que más se repite es el de dinamizador o guía. Sin embargo, existe la necesidad de profundizar en un mayor conocimiento del papel de las metodologías, el rol del docente en las mismas y el tipo de aprendizaje por parte del estudiante en el contexto de la Universidad Internacional de Valencia.

\author{
PALABRAS CLAVE: METODOLOGÍA, \\ DIDÁCTICA, UNIVERSIDAD EN LÍNEA, ROL \\ DOCENTE
}

\begin{abstract}
:
In every teaching-learning process, the methodologies determine the ways of working in the educational processes so that the students acquire the corresponding learning. It is a reality that marks the educational act and there are different modalities and roles conducted by the teacher in the development of them. Taking the Valencian International University as a reference in the context of online training, the objective of this study has been to analyze the use of methodologies in an online training context that were applied by 106 teachers who taught in the area of education during the 2017-2018 academic year. The data were collected through a self-administered questionnaire that was conducted ad hoc for this study. The questionnaire focused on four items: the methodologies that are implemented in the educational institution, the frequency of use, the role of the teacher when implementing the methodologies and the type of knowledge favoured in the student. The results show that regarding online learning most of the methodologies are used in a generalized way, except Hand-On methodology. The most common role of the teacher is the facilitator or guide role. However, there is a need to deepen in a greater knowledge concerning the role
\end{abstract}


of the methodologies, the role of the teacher applied therein and the type of learning performed by the student in the context of the Valencian International University.

KEYWORDS: METHODOLOGY, DIDACTICS, ONLINE UNIVERSITY, TEACHER ROLE

\section{INTRODUCCIÓN}

En el contexto formativo actual, la distancia y los horarios dejan de ser barreras con el aumento de la formación reglada en línea. Dentro de las diferentes instituciones que desarrollan este tipo de formación, la Universidad Internacional de Valencia (VIU) posee una oferta académica como universidad online que incluye grados y másteres oficiales, así como títulos propios.

Se caracteriza por una propuesta flexible e innovadora, ofreciendo una formación de calidad en línea construida sobre diferentes metodologías. El formato de clases permite al alumnado asistir en directo de manera síncrona o bien visualizarlas en diferido (tantas veces como desee) de manera asíncrona. De esta manera, los estudiantes pueden acceder desde cualquier lugar del mundo sin que la distancia suponga un obstáculo. A través de cualquier dispositivo móvil con conexión a la red, profesorado y alumnado pueden interactuar en tiempo real gracias a los diferentes recursos (sesiones, foro, correo, etc.).

Se rompe, siguiendo a Colomo y Aguilar (2017), con los obstáculos que un lugar físico y un horario concreto pueden generar, ampliando los escenarios de aprendizaje y favoreciendo así que los estudiantes puedan organizar su propio ritmo de aprendizaje en un proceso formativo en el que siempre están acompañados y guiados, siendo este uno de los beneficios de la era digital (Muñoz, Espiñeria y Rebollo, 2016; Rebollo y Espiñeira, 2015).

Respecto a los estudiantes, se trata de un perfil epresencial que se caracteriza por conjugar su vida profesional y personal con el deseo de seguir formándose (especializándose, capacitándose o habilitándose en muchos casos, en función de la titulación cursada) y aprendiendo en una realidad cada vez más competitiva y en constante cambio. Ante este prototipo de estudiantes, el reto reside en ofertar una educación de calidad y salvar los problemas que la presencialidad o semipresencialidad.

Por este motivo, la VIU se identifica con un carácter interactivo, en el que se trabaja constantemente en pos de la innovación educativa con el fin de poder implementar procesos de enseñanza-aprendizaje que se ajusten a las necesidades/requerimientos de sus estudiantes. La clave es favorecer la adquisición de competencias, habilidades y destrezas requeridas en la formación recibida de cara al ejercicio profesional.

Entre las diferentes áreas en las que se centran los procesos de innovación, cabe mencionar con especial relevancia el papel que las metodologías juegan en los procesos formativos de la VIU. Son la clave para alcanzar los retos propuestos para con los estudiantes, ya que determinan el modo de enseñanza sobre el cual se construirá su aprendizaje. En este aspecto, son varias las opciones metodológicas que se vienen implementando en la VIU, entre las que podemos señalar: aprendizaje colaborativo; trabajo en grupo; coevaluación; debate/foro; trabajo individual; proyecto; hand-on; juego de rol; estudio de casos; aprendizaje conceptual.

Tabla 1. Metodologías implementadas en la VIU. Elaboración propia.

\begin{tabular}{|c|c|}
\hline Metodologías & Descripción \\
\hline $\begin{array}{c}\text { Aprendizaje } \\
\text { colaborativo } \\
\text { (ACOL) }\end{array}$ & $\begin{array}{l}\text { Es una técnica didáctica que } \\
\text { promueve el aprendizaje centrado en } \\
\text { el alumno basando el trabajo en } \\
\text { pequeños grupos, donde los } \\
\text { estudiantes con diferentes niveles de } \\
\text { habilidad utilizan una variedad de } \\
\text { actividades de aprendizaje para } \\
\text { mejorar su entendimiento sobre una } \\
\text { materia. En el aprendizaje } \\
\text { colaborativo, el alumnado se } \\
\text { responsabiliza no solo de su } \\
\text { aprendizaje, sino también del de sus } \\
\text { compañeros, con el consiguiente } \\
\text { cambio de roles tanto en el } \\
\text { profesorado como en los estudiantes } \\
\text { (Avello y Marín, 2016). }\end{array}$ \\
\hline $\begin{array}{c}\text { Trabajo en } \\
\text { grupo } \\
\text { (TGRU) }\end{array}$ & $\begin{array}{l}\text { Es una metodología en la que } \\
\text { diferentes integrantes de un grupo } \\
\text { trabajan de forma conjunta en un } \\
\text { mismo fin, con una estructura } \\
\text { organizativa bien definida. Su } \\
\text { implementación potencia y favorece } \\
\text { las destrezas sociales, la } \\
\text { cooperación, la interacción y el } \\
\text { trabajo en } \\
\text { equipo (Gámez y Torres, 2013). }\end{array}$ \\
\hline $\begin{array}{c}\text { Coevaluación } \\
\text { (COEV) }\end{array}$ & $\begin{array}{l}\text { El foco reside en la evaluación por } \\
\text { pares entre el profesorado y el } \\
\text { alumnado sobre los trabajos que } \\
\text { estos van realizando. Vinculado a } \\
\text { ello, el estudiante incrementa su } \\
\text { autonomía y el feedback que obtiene } \\
\text { de su proceso de aprendizaje } \\
\text { (Barriopedro, López, Gómez y }\end{array}$ \\
\hline
\end{tabular}




\begin{tabular}{|c|c|}
\hline & Rivero, 2016). \\
\hline $\begin{array}{l}\text { Debate/Foro } \\
(\mathrm{D} / \mathrm{F})\end{array}$ & $\begin{array}{l}\text { Favorece la interacción entre los } \\
\text { diferentes agentes educativos sobre } \\
\text { las temáticas propuestas o que surjan } \\
\text { como fruto del intercambio de } \\
\text { opiniones. De esta forma, } \\
\text { potenciamos la reflexión y la } \\
\text { metacognición del estudiante (Basso, } \\
\text { Bravo, Castro y Moraga, 2018) }\end{array}$ \\
\hline $\begin{array}{l}\text { Trabajo } \\
\text { individual } \\
\text { (TIND) }\end{array}$ & $\begin{array}{l}\text { Se focaliza el proceso de enseñanza- } \\
\text { aprendizaje en el trabajo autónomo } \\
\text { por parte del estudiante, } \\
\text { enfrentándose de forma individual a } \\
\text { las acciones que se planteen y a la } \\
\text { resolución de las mismas. }\end{array}$ \\
\hline $\begin{array}{l}\text { Proyecto } \\
\text { (PRO) }\end{array}$ & $\begin{array}{l}\text { Método de enseñanza sustentado en } \\
\text { facilitar respuesta a un problema, } \\
\text { barrera o necesidad detectados en la } \\
\text { realidad o propuestos como reto. El } \\
\text { aprendizaje por proyectos sitúa al } \\
\text { alumno como protagonista del } \\
\text { proceso en el que el fin es aprender a } \\
\text { hacer y en el que el error es una fase } \\
\text { más en la adquisición de } \\
\text { conocimientos (Cuevas y Cívico, } \\
\text { 2018). }\end{array}$ \\
\hline $\begin{array}{l}\text { Hand-on } \\
(\mathrm{H}-\mathrm{ON})\end{array}$ & $\begin{array}{l}\text { Es una metodología activa y } \\
\text { experiencial que vincula el } \\
\text { aprendizaje a la acción, de manera } \\
\text { que el alumnado adquiera los } \\
\text { conocimientos mientras que los va } \\
\text { poniendo en práctica. }\end{array}$ \\
\hline $\begin{array}{l}\text { Juego de rol } \\
\quad(\text { JROL })\end{array}$ & $\begin{array}{l}\text { Mediante una dramatización de } \\
\text { casos reales o ficticios, el alumnado } \\
\text { puede interpretar diferentes papeles } \\
\text { y conocer la realidad desde las } \\
\text { diferentes perspectivas desde las que } \\
\text { se sitúe. Su potencial reside en el } \\
\text { valor educativo de dos herramientas, } \\
\text { como el diálogo y el razonamiento, } \\
\text { que permiten el desarrollo y ejercicio } \\
\text { del pensamiento crítico del } \\
\text { estudiante (Colomo y Cívico, 2018). }\end{array}$ \\
\hline $\begin{array}{l}\text { Estudio de } \\
\text { casos } \\
\text { (ECAS) }\end{array}$ & $\begin{array}{l}\text { Metodología que permite al } \\
\text { alumnado conocer y comprender las } \\
\text { casuísticas de un contexto con el fin } \\
\text { de que pueda analizar y comprender } \\
\text { cómo funcionan los distintos } \\
\text { elementos y las relaciones que se } \\
\text { generan entre ellos. El objetivo final } \\
\text { de los estudios de casos es } \\
\text { profundizar en las particularidades, } \\
\text { no tratándose de ver en qué se } \\
\text { diferencia de otro sino en saber cuál } \\
\text { es la realidad del caso concreto, qué } \\
\text { se hace (Stake, 2005). }\end{array}$ \\
\hline $\begin{array}{l}\text { Aprendizaje } \\
\text { conceptual } \\
(\mathrm{ACON})\end{array}$ & $\begin{array}{l}\text { Metodología vinculada a la } \\
\text { reestructuración de esquemas } \\
\text { cognitivos sobre la realidad a partir } \\
\text { de la información y contenidos que }\end{array}$ \\
\hline
\end{tabular}
se van abordando. La adquisición de los conceptos de forma significativa es clave ya que fundamentarán las conexiones futuras que se realicen a nivel cognitivo.

Por otro lado, si atendemos al rol docente que se desprende de la acción formativa de los profesores en la VIA, apuntaban Gabarda, Rodríguez y Romero (2016) que esta se centraba en dos ejes fundamentales:

- La docencia: estructuración de las actividades, dominio de la materia, exposición de los resultados de aprendizaje, adecuación de los materiales, explicación de las actividades y utilización de los recursos.

- La tutorización y atención, la motivación y participación: seguimiento individualizado, motivación y fomento de la participación y de las relaciones interpersonales.

Partiendo de esta realidad, el objetivo principal al que nos dirigimos es analizar el uso de las metodologías que se aplican en un contexto de formación en línea. Este objetivo general se subdivide en varios específicos, entre los que se encuentran: a) conocer el grado de implementación y la frecuencia de uso de diferentes metodologías en la institución objeto de estudio; b) analizar el papel del docente en el desarrollo de cada una de las metodologías; c) profundizar sobre los tipos de aprendizaje que se asocian a cada metodología.

\section{MATERIAL Y MÉTODO}

\subsection{Participantes}

La población objeto de estudio está conformada por 321 profesores de la VIU que imparten docencia en el área de Educación en el curso 2017-2018.

La totalidad de la muestra consta de 106 profesores $(\mathrm{N}=106)$, que representan un $33 \%$ de la población. En dicha muestra, un $76,4 \%$ son mujeres (81) y un $23,6 \%$ son hombres (25), con una edad comprendida entre los 25 y 64 años $(\mathrm{Md}=37,8)$.

En el presente estudio se utilizó un diseño trasversal de tipo descriptivo. La muestra fue seleccionada a través de un muestreo no probabilístico, del tipo de conveniencia o causal (Alaminos, 2006; Sabariego, 2012) que se basa en la facilidad de acceso por parte del investigador a los participantes del estudio. En este caso los investigadores eran profesores de la misma área en que se realiza el estudio (Educación). 


\subsection{Procedimiento}

El criterio para la selección de los sujetos de la muestra de estudio fue la cumplimentación de un cuestionario autoadministrado de manera voluntaria y anónima, a través de la aplicación Google Forms (online), por su flexibilidad y capacidad para ajustarse a las necesidades de la investigación de forma gratuita y sin limitaciones (Abundis, 2016).

Previamente, se informó al claustro sobre el estudio que se estaba diseñando, con objeto de dar información específica al respecto y solicitando la colaboración.

\subsection{Instrumento}

El instrumento ha sido diseñado ad hoc para esta investigación, estando compuesto de dos partes diferenciadas. La primera de ellas se centra en conocer algunas características personales del profesorado y que recogía información de variables como el sexo (hombre/mujer), la edad y las titulaciones en que impartía docencia.

La segunda parte de la encuesta se compone de cuatro ítems, orientados a conocer cuáles de las metodologías expuestas se utilizan en la institución (aprendizaje colaborativo, trabajo en grupo, coevaluación, debate/foro, trabajo individual, proyecto, hand-on, juego de rol, estudio de caso, aprendizaje conceptual), con qué frecuencia, qué tipo de rol ejerce el docente en cada metodología y qué tipo de conocimiento favorece en el estudiante.

Se ofrece a continuación el detalle de los ítems y las alternativas de respuesta ofrecidas en cada uno de ellos:

Tabla 2. Ítems y alternativas de respuesta. Elaboración propia.

\begin{tabular}{|c|c|}
\hline Ítem & Alternativas de respuesta \\
\hline $\begin{array}{l}\text { ¿La institución } \\
\text { utiliza este } \\
\text { método? }\end{array}$ & Sí / No / No lo sé \\
\hline $\begin{array}{l}\text { ¿Cuándo utiliza } \\
\text { este método? }\end{array}$ & $\begin{array}{l}\text { Siempre / Algunas veces / Casi } \\
\text { nunca / } \\
\text { Nunca / No lo sé }\end{array}$ \\
\hline $\begin{array}{l}\text { ¿Qué rol } \\
\text { fundamental } \\
\text { debe } \\
\text { desarrollar el } \\
\text { docente? }\end{array}$ & $\begin{array}{l}\text { Transmisor de la información / } \\
\text { Planificador / Dinamizador / } \\
\text { Guía / No conozco el método }\end{array}$ \\
\hline $\begin{array}{l}\text { ¿Qué tipo de } \\
\text { conocimiento } \\
\text { favorece en el } \\
\text { estudiante? }\end{array}$ & $\begin{array}{ll}\text { Conocimiento } & \text { del } \\
\text { procedimientos / Conocimiento } \\
\text { de conceptos / Conocimiento } \\
\text { aplicado / Conocimiento } \\
\text { teórico / Resolución de } \\
\text { problemas prácticos / No } \\
\text { conozco el método }\end{array}$ \\
\hline
\end{tabular}

\subsection{Análisis de datos}

Mediante el paquete estadístico IBM SPSS Statistics 20 se llevaron a cabo análisis descriptivos de las puntuaciones de los ítems que componen el cuestionario, los cuales se muestra a continuación en el apartado de resultados.

\section{RESULTADOS}

Tal y como apuntábamos anteriormente, los ítems de la encuesta se orientaban a conocer si se utilizaban en la institución las metodologías explicadas con anterioridad, con qué frecuencia, qué rol ejercían los profesores con cada una de ellas y qué tipo de aprendizaje suponía para los estudiantes.

En relación a la primera pregunta, y tal y como puede observarse en la figura 1, el $100 \%$ del profesorado manifestaba que la institución hacía uso del trabajo individual. Porcentajes superiores al $90 \%$ pueden observarse en el debate/foro $(98,1 \%)$, el aprendizaje conceptual $(92,5 \%)$ y el trabajo en grupo $(91,5 \%)$.

Por otro lado, el 87,7\% afirman que la institución utiliza el estudio de caso, un 78,3\% el aprendizaje colaborativo y un $76,4 \%$ el proyecto.

Por último, las metodologías menos utilizadas por la institución según la percepción de los profesores serían la coevaluación $(48,1 \%)$, el juego de rol $(34,0 \%)$ y el hand-on $(22,6 \%)$.

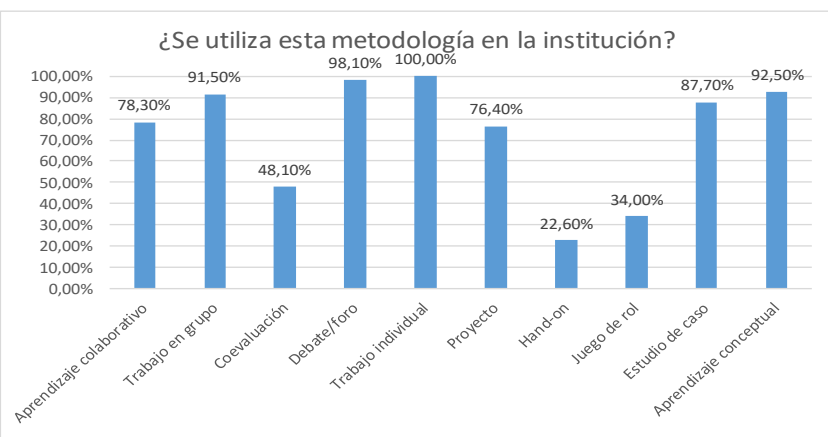

Figura 1. Utilización de las metodologías por parte de la institución. Elaboración propia

En relación a la frecuencia con que se utiliza cada una de las metodologías, los resultados arrojan que todas las propuestas son utilizadas "siempre" o "algunas veces", habiendo puntuaciones muy bajas en metodologías que no se utilizan (ver tabla 3).

Concretamente, los docentes manifiestan utilizar siempre el trabajo individual $(70,8 \%)$, debate/foro (67\%), el aprendizaje conceptual $(57,8 \%)$, el trabajo en grupo $(47,2 \%)$ y el aprendizaje colaborativo (41,5\%). 
Tabla 3. Frecuencia de uso de cada metodología. Elaboración propia.

\begin{tabular}{cccccc}
\hline $\begin{array}{c}\text { Metodo } \\
\text {-logía }\end{array}$ & $\begin{array}{c}\text { Siem- } \\
\text { pre }\end{array}$ & $\begin{array}{c}\text { Algu- } \\
\text { nas } \\
\text { veces }\end{array}$ & $\begin{array}{c}\text { Casi } \\
\text { nunca }\end{array}$ & No & No lo sé \\
\hline ACOL & $41,51 \%$ & $41,51 \%$ & $0,00 \%$ & $0,94 \%$ & $16,04 \%$ \\
\hline TGRU & $47,17 \%$ & $44,34 \%$ & $0,94 \%$ & $0,94 \%$ & $6,60 \%$ \\
\hline COEV & $12,26 \%$ & $37,74 \%$ & $11,32 \%$ & $10,38 \%$ & $28,30 \%$ \\
\hline D/F & $66,98 \%$ & $27,36 \%$ & $2,83 \%$ & $0,94 \%$ & $1,89 \%$ \\
\hline TIND & $70,75 \%$ & $27,36 \%$ & $0,00 \%$ & $0,00 \%$ & $1,89 \%$ \\
\hline PRO & $21,70 \%$ & $53,77 \%$ & $3,77 \%$ & $6,60 \%$ & $14,15 \%$ \\
\hline H-ON & $5,66 \%$ & $13,21 \%$ & $2,83 \%$ & $3,77 \%$ & $74,53 \%$ \\
\hline JROL & $1,89 \%$ & $33,02 \%$ & $9,43 \%$ & $15,09 \%$ & $40,57 \%$ \\
\hline ECAS & $16,98 \%$ & $66,04 \%$ & $6,60 \%$ & $0,94 \%$ & $9,43 \%$ \\
\hline ACON & $57,55 \%$ & $35,85 \%$ & $0,00 \%$ & $0,00 \%$ & $6,60 \%$ \\
\hline
\end{tabular}

Concretamente, los docentes manifiestan utilizar siempre el trabajo individual $(70,8 \%)$, debate/foro (67\%), el aprendizaje conceptual (57,8\%), el trabajo en grupo $(47,2 \%)$ y el aprendizaje colaborativo $(41,5 \%)$.

Estas mismas metodologías obtienen también puntuaciones altas en la frecuencia "algunas veces": aprendizaje individual $(27,36 \%)$, debate/foro $(27,36 \%)$, aprendizaje conceptual $(35,85 \%)$, trabajo en grupo $(44,34 \%)$ y aprendizaje colaborativo $(41,51 \%)$.

Al margen de estas metodologías, se utilizan "algunas veces" el estudio de caso $(66,34 \%)$, el proyecto $(53,77 \%)$ y la coevaluación $(37,74 \%)$, que son utilizadas "siempre" según un $16,98 \%$, un $21,7 \%$ y un $12,26 \%$ del profesorado respectivamente.

Por último, cabe resaltar que no saben con qué frecuencia se utiliza el hand-on un $74,5 \%$ de los profesores, habiendo un dato del $40,6 \%$ en el caso del juego de rol.

En relación al tipo de rol que debe desarrollar el docente con cada una de las metodologías, hay una identificación clara de que el foro/debate requiere que el docente desarrolle el rol de dinamizador $(73,6 \%)$ y la relación entre el aprendizaje conceptual y el rol de transmisor de la información (67\%), (ver tabla 4).

Como puede observarse, hay metodologías como el aprendizaje colaborativo, el trabajo en grupo y el juego de rol donde el profesorado identifica que el rol docente es predominantemente de dinamizador y guía, siendo escaso el porcentaje que identifica roles de transmisor de información o planificador. Este rol se asocia, por tanto, a actividades de carácter eminentemente práctico.
Tabla 4. Rol docente por metodología. Elaboración propia.

\begin{tabular}{|c|c|c|c|c|c|}
\hline $\begin{array}{c}\text { Meto- } \\
\text { dología }\end{array}$ & $\begin{array}{l}\text { Trans- } \\
\text { misor } \\
\text { de la } \\
\text { infor- } \\
\text { mación }\end{array}$ & $\begin{array}{l}\text { Planifi- } \\
\text { cador }\end{array}$ & $\begin{array}{l}\text { Dinami } \\
\text {-zador }\end{array}$ & Guía & $\begin{array}{c}\text { No } \\
\text { conozco }\end{array}$ \\
\hline ACOL & $6,60 \%$ & $12,26 \%$ & $41,51 \%$ & $37,74 \%$ & $1,89 \%$ \\
\hline TGRU & $9,43 \%$ & $11,32 \%$ & $39,62 \%$ & $39,62 \%$ & $0,00 \%$ \\
\hline COEV & $12,26 \%$ & $21,70 \%$ & $25,47 \%$ & $25,47 \%$ & $15,09 \%$ \\
\hline $\mathrm{D} / \mathrm{F}$ & $3,77 \%$ & $11,32 \%$ & $73,58 \%$ & $11,32 \%$ & $0,00 \%$ \\
\hline TIND & $34,91 \%$ & $11,32 \%$ & $7,55 \%$ & $46,23 \%$ & $0,00 \%$ \\
\hline PRO & $3,77 \%$ & $29,25 \%$ & $12,26 \%$ & $51,89 \%$ & $2,83 \%$ \\
\hline $\mathrm{H}-\mathrm{ON}$ & $0,94 \%$ & $14,15 \%$ & $15,09 \%$ & $6,60 \%$ & $63,21 \%$ \\
\hline JROL & $0,00 \%$ & $18,87 \%$ & $37,74 \%$ & $21,70 \%$ & $21,70 \%$ \\
\hline ECAS & $20,75 \%$ & $16,04 \%$ & $14,15 \%$ & $47,17 \%$ & $1,89 \%$ \\
\hline ACON & $66,98 \%$ & $4,72 \%$ & $4,72 \%$ & $20,75 \%$ & $2,83 \%$ \\
\hline
\end{tabular}

Por otro lado, en el estudio de caso y el trabajo individual, el profesorado identifica como rol docente predominante el de guía y el de transmisor de la información. Se trata, por tanto, de metodologías donde el docente cumple un doble papel, exponiendo la información básica necesaria para afrontar una tarea en la que el profesorado cambia de rol.

En el caso del proyecto, se concibe que el docente ha de cumplir con los roles de guía y planificador, ejerciendo un papel clave para la preparación y el desarrollo de la actividad. Mayor dispersión existe entre las puntuaciones sobre el rol docente en la coevaluación que, según el profesorado, ejercería de dinamizador, guía y planificador. Por último, y como viene observándose en análisis previos, hay un desconocimiento generalizado sobre la metodología del hand-on. En este sentido, el $63,2 \%$ del profesorado manifiesta no conocer cuál sería el rol docente en esta metodología.

El último ítem se orienta a conocer qué tipo de aprendizaje promueve entre los estudiantes cada metodología, según la percepción del profesorado (ver tabla 5).

Tabla 5. Tipo de aprendizaje por metodología. Elaboración propia

\begin{tabular}{lcccccc}
\hline & $\begin{array}{c}\text { Conoci- } \\
\text { miento } \\
\text { del } \\
\text { procedi } \\
\text { mien- } \\
\text { tos }\end{array}$ & $\begin{array}{c}\text { Conoci- } \\
\text { miento } \\
\text { de } \\
\text { concep- } \\
\text { tos }\end{array}$ & $\begin{array}{c}\text { Conoci- } \\
\text { miento } \\
\text { aplica- } \\
\text { do }\end{array}$ & $\begin{array}{c}\text { Conoci- } \\
\text { miento } \\
\text { teórico }\end{array}$ & $\begin{array}{c}\text { Resolu- } \\
\text { ción de } \\
\text { proble- } \\
\text { mas } \\
\text { prácti- } \\
\text { cos }\end{array}$ & $\begin{array}{c}\text { No } \\
\text { conoz- } \\
\text { co el } \\
\text { método }\end{array}$ \\
\hline ACOL & $19,81 \%$ & $3,77 \%$ & $33,96 \%$ & $1,89 \%$ & $38,68 \%$ & $1,89 \%$ \\
\hline TGRU & $11,32 \%$ & $5,66 \%$ & $50,00 \%$ & $1,89 \%$ & $31,13 \%$ & $0,00 \%$ \\
\hline COEV & $18,87 \%$ & $12,26 \%$ & $29,25 \%$ & $12,26 \%$ & $10,38 \%$ & $16,98 \%$ \\
\hline D/F & $7,55 \%$ & $20,75 \%$ & $41,51 \%$ & $13,21 \%$ & $16,98 \%$ & $0,00 \%$ \\
\hline TIND & $10,38 \%$ & $35,85 \%$ & $24,53 \%$ & $25,47 \%$ & $3,77 \%$ & $0,00 \%$ \\
\hline
\end{tabular}




\begin{tabular}{lrrrrrr}
\hline PRO & $11,32 \%$ & $5,66 \%$ & $50,00 \%$ & $3,77 \%$ & $25,47 \%$ & $3,77 \%$ \\
\hline H-ON & $2,83 \%$ & $0,00 \%$ & $18,87 \%$ & $0,00 \%$ & $10,38 \%$ & $67,92 \%$ \\
\hline JROL & $2,83 \%$ & $1,89 \%$ & $32,08 \%$ & $0,00 \%$ & $38,68 \%$ & $24,53 \%$ \\
\hline ECAS & $11,32 \%$ & $1,89 \%$ & $38,68 \%$ & $5,66 \%$ & $38,68 \%$ & $3,77 \%$ \\
\hline ACON & $1,89 \%$ & $69,81 \%$ & $4,72 \%$ & $20,75 \%$ & $0,00 \%$ & $2,83 \%$ \\
\hline
\end{tabular}

\begin{tabular}{|c|c|c|c|}
\hline $\mathrm{H}-\mathrm{ON}$ & No lo sé & No lo sé & No lo sé \\
\hline JROL & No lo sé & Dinamizador & $\begin{array}{l}\text { Resolución de } \\
\text { problemas } \\
\text { prácticos }\end{array}$ \\
\hline ECAS & $\begin{array}{c}\text { Algunas } \\
\text { veces }\end{array}$ & Guía & $\begin{array}{l}\text { Conocimiento } \\
\text { aplicado/ } \\
\text { Resolución de } \\
\text { problemas } \\
\text { prácticos }\end{array}$ \\
\hline $\mathrm{ACON}$ & Siempre & $\begin{array}{c}\text { Transmisor } \\
\text { de la } \\
\text { información }\end{array}$ & $\begin{array}{c}\text { Conocimiento } \\
\text { de conceptos }\end{array}$ \\
\hline
\end{tabular}

En este sentido, alrededor de tres de cada cuatro docentes identifican que el aprendizaje colaborativo, el trabajo en grupo, el proyecto, el juego de rol y el estudio de caso se asocian a un conocimiento aplicado y de resolución de problemas prácticos.

En contraposición, consideran que el aprendizaje conceptual se asocia al conocimiento de conceptos $(69,81 \%)$ y al conocimiento teórico $(20,75 \%)$. El trabajo individual, por otro lado, promueve el conocimiento de conceptos $(35,85 \%)$, el conocimiento aplicado $(24,53 \%)$ y el conocimiento teórico $(25,47 \%)$. Esta metodología, junto a la coevaluación, son las que se identifican con una mayor variedad de tipos de aprendizaje. En el caso de esta última se asocia de manera repartida al conocimiento aplicado, el conocimiento de procedimientos, el conocimiento de conceptos y el conocimiento teórico.

Por otro lado, el debate/foro se identifica con un aprendizaje de conocimiento aplicado y de conocimiento de conceptos, viéndose de este modo el carácter teórico-práctico de la metodología. Por último, un $67,92 \%$ de los docentes afirman no conocer qué tipo de aprendizaje se asocia al handon.

A modo de resumen, se detalla a continuación la frecuencia de uso más habitual de cada una de las metodologías, así como el rol predominante que juega el docente y el tipo de aprendizaje para el estudiante al que se asocia:

Tabla 6. Metodología, frecuencia de uso, rol docente predominante y tipo de aprendizaje más habitual. Elaboración propia

\begin{tabular}{|c|c|c|c|}
\hline Metodología & $\begin{array}{c}\text { Frecuencia } \\
\text { de uso }\end{array}$ & Rol docente & $\begin{array}{c}\text { Tipo de } \\
\text { aprendizaje }\end{array}$ \\
\hline ACOL & $\begin{array}{c}\text { Siempre/ } \\
\text { Algunas } \\
\text { veces }\end{array}$ & Dinamizador & $\begin{array}{l}\text { Resolución de } \\
\text { problemas } \\
\text { prácticos }\end{array}$ \\
\hline TGRU & $\begin{array}{c}\text { Siempre/ } \\
\text { Algunas } \\
\text { veces }\end{array}$ & $\begin{array}{c}\text { Dinamizador } \\
\text { / Guía }\end{array}$ & $\begin{array}{c}\text { Conocimiento } \\
\text { aplicado }\end{array}$ \\
\hline COEV & $\begin{array}{l}\text { Algunas } \\
\text { veces }\end{array}$ & $\begin{array}{c}\text { Dinamizador } \\
\text { / Guía }\end{array}$ & $\begin{array}{c}\text { Conocimiento } \\
\text { aplicado }\end{array}$ \\
\hline $\mathrm{D} / \mathrm{F}$ & Siempre & Dinamizador & $\begin{array}{c}\text { Conocimiento } \\
\text { aplicado }\end{array}$ \\
\hline TIND & Siempre & Guía & $\begin{array}{c}\text { Conocimiento } \\
\text { de conceptos }\end{array}$ \\
\hline PRO & $\begin{array}{c}\text { Algunas } \\
\text { veces }\end{array}$ & Guía & $\begin{array}{c}\text { Conocimiento } \\
\text { aplicado }\end{array}$ \\
\hline
\end{tabular}

\section{DISCUSIÓN Y CONCLUSIONES}

Analizando el cumplimiento del objetivo general del estudio, éste se dirigía a analizar el uso de las metodologías que se aplican en un contexto de formación en línea.

En este sentido, hemos constatado que las diferentes metodologías propuestas se utilizan de manera generalizada, a excepción de la coevaluación, el hand-on y el juego de rol. Como hemos recogido en la fundamentación teórica, las metodologías juegan un papel clave al determinar las formas de trabajar en los procesos de enseñanza para que el alumnado adquiera los correspondientes aprendizajes. Por este motivo, la variedad y el conocimiento de las bases de la misma se hace preciso para ofrecer una formación de calidad.

Respecto al primer objetivo específico (conocer el grado de implementación y la frecuencia de uso de diferentes metodologías en la institución objeto de estudio), se concluye que la mayor parte de las metodologías propuestas se utilizan "siempre" o "algunas veces". Las excepciones serían el hand-on y el juego de rol, cuya frecuencia de uso se desconoce por parte del profesorado.

De hecho, el 22,6\% del profesorado que han conformado la muestra objeto de estudio afirman que la metodología hand-on se utiliza en la VIU, es de destacar cómo el 67,92\% no conoce este tipo de aprendizaje, el $63,21 \%$ no conoce el rol que debe desempeñar el docente y el 74,53\% no conoce la frecuencia de su uso en la institución. Estos datos contrastan con una metodología que se caracteriza por fomentar un aprendizaje eminentemente práctico, un aspecto fundamental a considerar en la formación en línea.

En el caso de la metodología de juego de roles, el $34 \%$ de los docentes afirman que esta metodología se utiliza en VIU. Sin embargo, el $24,53 \%$ no conoce este tipo de aprendizaje y un 21,7\% tampoco sabe el rol que debe desempeñar el docente. Como sucedió con el hand-on, los datos no tienen relación 
con una metodología como el juego de roles en la que el diálogo y el razonamiento, aspectos fundamentales en un proceso de aprendizaje constructivista, son el eje que permite el desarrollo del alumnado.

$\mathrm{Si}$ atendemos, por último, a los objetivos específicos relativos a analizar el papel del docente en el desarrollo de cada una de las metodologías y profundizar sobre los tipos de aprendizaje que se vinculan a cada metodología, los datos arrojan información relevante para el estudio.

De manera generalizada, el rol docente que se asocia a las diferentes metodologías es el de dinamizador o de guía. Las excepciones son de nuevo el hand-on, cuyo rol docente se desconoce por parte de los encuestados, y el aprendizaje conceptual, que se asocia a un rol de transmisor de la información.

Si contraponemos la percepción que tiene el profesorado acerca del rol docente que ejerce el docente en cada metodología y el tipo de aprendizaje que favorece con la literatura previa, observamos algunas coincidencias, pero también algunas discrepancias subrayables.

Apuntaban Avello y Marín (2016) que el potencial del aprendizaje colaborativo se centra en el alumnado y en el grupo, así como el cambio de roles de profesor y estudiante. Esta propuesta guardaría relación con la percepción de los participantes en este estudio, que coincidían mayoritariamente en el papel dinamizador del docente, alejándose de la transmisión de la información.

No habría coincidencia, sin embargo, en la concepción de la coevaluación. Mientras Barriopedro et al. (2016) destacaban la reflexión y metacognición del estudiante $\mathrm{y}$, por tanto, su carácter conceptual, se pone de manifiesto en este estudio que el profesorado lo asocia a un tipo de aprendizaje aplicado.

Similar es la discrepancia entre la propuesta de Colomo y Cívico (2018) acerca del juego de rol, en el que destacaban su potencial para el diálogo y el razonamiento, mientras que el profesorado encuestado lo identifica con un tipo de aprendizaje eminentemente aplicado.

Como hemos podido observar en nuestro caso de estudio, el papel de las metodologías, el rol del docente en las mismas y el tipo de aprendizaje por parte del estudiante son aspectos a seguir trabajando y perfeccionado en el contexto de la VIU.

Pese a que la libertad de cátedra flexibilizará la elección por parte de cada docente del uso de la metodología más adecuada en función del tipo de aprendizaje que se desee desarrollar, los resultados nos indican la necesidad de profundizar en un mayor conocimiento por parte del profesorado, pudiendo articular acciones formativas en este sentido.

\section{REFERENCIAS}

Abundis, V. (2016). Beneficios de las encuestas electrónicas como apoyo para la investigación. Tlatemoani. Revista Académica de Investigación, 22, 168-186.

Alaminos, A. (2006). El muestreo en la investigación social. En A. Alaminos y J.L. Castejón. Elaboración, análisis e interpretación de encuestas, cuestionarios y escalas de opinión (pp. 46-67). Alcoy: Marfil.

Avello, R., Marín, V. I. (2016). La necesaria formación de los docentes en aprendizaje colaborativo. Profesorado. Revista de Currículum y Formación de Profesorado, 20(3), 687-713.

Barriopedro, M., López, C., Gómez, M.Á., y Rivero, A. (2016). La coevaluación como estrategia para mejorar la dinámica del trabajo en grupo: una experiencia en Ciencias del Deporte. Revista Complutense de Educación, 27(2), 571-584.

Basso, M., Bravo, M., Castro, A., y Moraga, C. (2018). Propuesta de modelo tecnológico para Flipped Classroom (T-FliC) en educación superior. Revista Electrónica Educare (Educare Electronic Journal), 22(2), 1-17.

Colomo, E., y Aguilar, Á.I. (2017). Píldoras formativas en la educación online: posibilidades y limitaciones. En J. Ruiz-Palmero, J. Sánchez-Rodríguez, y E. SánchezRivas (Edit.). Innovación docente y uso de las TIC en educación. Málaga: UMA editorial.

Colomo, E., y Cívico, A. (2018). La necesidad de formación del profesorado en pedagogía de la muerte. Revista Electrónica Interuniversitaria de Formación del Profesorado, 21(1), 83-94. doi: http://dx.doi.org/10.6018/reifop.21.1.279961

Cuevas, N., y Cívico, A. (2018). El desarrollo de las competencias básicas mediante el aprendizaje por proyectos. Harvad Deusto: Learning \& Pedagogics, 14, 30-35.

Gabarda, V., Rodríguez, A., y Romero, M. M. (2016). Percepción del alumnado en procesos de tutorización en educación superior online. Revista Opción, 32(7), 630644.

Gámez, M. J., y Torres, C. (2013). Las técnicas de grupo como estrategia metodológica en la adquisición de la competencia de trabajo en equipo de los alumnos universitarios. Journal for Educators, Teachers and Trainers, 4(1), 14-25.

Muñoz, J.M., Espiñeira, E.M. y Rebollo, N. (2016). Las píldoras formativas: diseño y desarrollo de un modelo de evaluación en el Espacio Europeo Superior en Educación Superior. Revista de investigación en educación, 2(14), 156-169.

Rebollo, N. y Espiñeira, E.M. (2015). Una alternativa complementaria a la formación: las píldoras. Revista de estudios e investigación en psicología de la educación, Extra (10), 91-94.

Sabariego, M. (2012). El proceso de investigación. En R. Bisquerra (Coord.). Metodología de la investigación educativa (pp. 127 - 163). Madrid: La Muralla.

Stake, R. (2005). Qualitative case studies. En N. Denzin, y Y. Lincoln (Ed.) The Sage handbook of qualitative research. Thousand Oaks: Sage. 